\title{
A novel type II relay-assisted retransmission scheme for uplink of LTE-advanced system
}

\author{
Anxin $\mathrm{Li}^{1 *}$, Satoshi Nagata ${ }^{2}$, Atsushi Harada ${ }^{1}$ and Hirohito Suda
}

\begin{abstract}
Relay, which enables coverage extension and throughput enhancement, is a very promising technique for future wireless communication systems. Among different types of relay, type II relay is one kind of inband relays and is hotly discussed in LTE-Advanced system for throughput enhancement. In order to support type II relay, many challenges must be overcome. In this article, we focus on relay-assisted uplink data retransmission and propose a novel joint design of reference signal and data precoding for type II relay. The proposed method not only solves the problem of channel estimation mismatch for control information, but also achieves cooperative diversity gain for data transmission. Simulation results show the effectiveness of the proposed method over existing schemes.
\end{abstract}

Keywords: Relay, Transparent relaying, Data retransmission, Channel estimation mismatch

\section{Introduction}

Relay is a very promising technique for future wireless communication systems to extend coverage and enhance throughput, and has drawn increasing interest from both academia and industry [1-6]. There are many types of relays and some of them have been specified for future mobile systems, such as WiMax and LTE-Advanced system [5]. Two types of relays, i.e., type I and type II, are mainly discussed for LTE-advanced systems in the 3rd Generation Partnership Project (3GPP) [3,7-9]. The type I relay is a kind of non-transparent relay with wireless backhaul to the donor eNodeB cell. The type I relay terminates all layers 2 and 3 protocols and appears as a normal eNodeB to user equipment (UE). According to the backhaul, type I relay can be classified into inband (same frequency band for backhaul link and access link) and half-duplex (backhaul transmission and access transmission at different time), inband and full-duplex (backhaul transmission and access transmission at same time), and outband (different frequency band for backhaul link and access link). The type I relay has its own cell and physical cell identification (ID). It transmits synchronization signals and performs resource allocation. The typical usage scenarios of type I relay include coverage extension, e.g., hotspot and

\footnotetext{
* Correspondence: liax@docomolabs-beijing.com.cn

'DOCOMO Beijing Communications Laboratories Co, Ltd, 7/F, Raycom Infotech Park Tower A, No. 2 Kexueyuan South Road, Haidian District, Beijing 100190, China

Full list of author information is available at the end of the article
}

deadspot, and group mobility, e.g., on a train or bus. On the other hand, the type II relay is a kind of inband transparent relay and works in a half-duplex mode. Usually, the type II relay only supports functions up to layer 2 . Type II relay does not have a physical cell ID and is a part of a donor eNodeB cell. The eNodeB directly controls the UEs in the cell and performs resource allocation. The type II relay transparently participates in data forwarding in downlink and uplink, i.e., the UE is not aware of whether or not it is communicating with the eNodeB via the relay. As a result, the type II relay should not transmit synchronization signal, common reference signal, etc. Therefore, the type II relay cannot be used for coverage extension and the main purpose of the type II relay is to enhance throughput and quality-of-service of UEs, especially for cell-edge UEs, in the macro cell.

Although there have been a lot of research works about type I relay, the research works about type II relay are just started and relatively not enough. Type II relay has many advantages compared with type I relay, such as low cost (layer 2 relay), easy of deployment (transparent), and good throughput enhancement (cooperative transmission with eNodeB in downlink and UEs in uplink in nature). However, in order to exploit the benefits and potentials of the type II relay, more sophisticated designs are needed than type I relay. The transparency and backward compatibility requirement bring many challenges facing the implementation of the type II relay in LTE-Advanced 
systems $[3,6,10]$. Overall speaking, two issues of the type II relay are of most importance. One is the type II relay-assisted cooperative hybrid automatic repeat request (HARQ) [3] and the other is link adaptation for type II relay-assisted data transmission. In this article, we focus on an enhanced type II relay-assisted uplink data retransmission scheme in order to improve block error rate (BLER) performance while maintaining backward compatibility to legacy UEs.

One major problem in using the type II relay in uplink data retransmission $[10,11]$ is caused by the backward compatibility requirement. As specified in LTE-Advanced specifications, when a legacy UE has both control information (e.g., ACK/NACK, periodic channel quality indicator (CQI) report, etc.), and data to be transmitted in the uplink, the UE should embed the control information in the data transmission in order to retain the single carrier-frequency division multiple access characteristics and to reduce peak-to-average power ratio (PAPR). Such a transmission requirement, i.e., embedding control information in data, brings difficulty for type II relay-assisted cooperative retransmission, where type II relay monitors initial transmission of the UE and performs cooperative transmission to assist retransmission of UE when the initial transmission is failed. We assume that the UE has control information (e.g., ACK/NACK to different downlink data packets) to be transmitted at both initial transmission and retransmission. For simplicity, we denote the control information embedded in initial transmission as "old" control information and the control information embedded in retransmission as "new" control information. The type II relay can only know the old control information through monitoring and detecting the initial transmission but cannot know the new control information to be transmitted by the UE in advance before the retransmission. Therefore, type II relay will have difficulty in performing the retransmission. If the type II relay sends exactly the same signals as the initial transmission of the UE, i.e., with the old control information, the old control information from the relay and the new control information from the UE will collide at the eNodeB. If the relay punctures the old control information in cooperative retransmission, the eNodeB receives the control information only from the UE but a superposed reference signal from both the relay and the UE. Consequently, channel estimation mismatch occurs for the control information and results in unacceptable detection performance of the control information.

In order to address this problem, some methods have been proposed in $[12,13]$. In the method in [12], the eNodeB always sends an ACK to the UE no matter whether data detection is correct or not. In case the detection at the eNodeB is not correct, the ACK is a fake one. The purpose to always transmit ACK to the
UE is to make UE think the data transmission is successful. Because the ACK is received, the UE does not need to retransmit data and thus the control information is transmitted in control channel rather than being embedded in the data channel. Only the relay performs data retransmission, i.e., transmits the data and reference signal in the data channel. Because the control channel has its own reference signal, there is no channel estimation mismatch of control information any longer. However, there are several problems with the method: (1) New signaling is required, e.g., eNodeB must inform the relay of retransmission because the relay monitors a fake ACK and cannot make a decision based on it. (2) If the relay does not correctly receive a data packet and the UE clears its buffer after receiving the ACK, the data packet may not be recovered by HARQ and thus may cause a long delay. (3) The UE occupies additional control channel resource to transmit control information. (4) Only relay performs the retransmission and thus there is no cooperative diversity gain in data transmission. In [13], two methods are proposed. In the first method, the relay reports its detection result of initial transmission to eNodeB. If the relay correctly detects the data of UE, eNodeB will send a fake ACK to the UE in order to stop retransmission of the UE. Instead, relay will conduct the retransmission to send the data to eNodeB. Although the above HARQ failure problem can be solved by this method, the other drawbacks still exits. (1) Additional signaling is needed, e.g., the relay needs to inform eNodeB of its data detection result. (2) The UE occupies additional control channel resource to transmit control information. Because the relay conducts the retransmissions, if the UE has new control information to report to eNodeB, the UE has to transmit the new control information in control channel. (3) There is no cooperative diversity gain because either the UE or the relay conducts retransmission. In the second method, after initial transmission of the UE, eNodeB always sends an ACK (maybe fake) to the UE regardless the data detection is correct or not. At the same time, eNodeB sends the actual data detection result to the relay. If the data detection at eNodeB is not correct, the relay will conduct retransmission to eNodeB. This method is the same as the "Always ACK" method in [12] except that the UE and the relay can select modulation and coding scheme used for transmission according to quality of UE-to -relay channel and relay-to-eNodeB channel, respectively, and thus may improve the spectrum efficiency of the transmission/retransmission. For the second method, all the drawbacks of "Always ACK" method exist.

In this article, we propose a novel retransmission method that is fully backward compatible to legacy UEs without any new signaling, does not require additional control channel resource for control information, has no 
HARQ failure problem, and achieves cooperative diversity gain for data retransmission. The major contributions of the article lie in two aspects. One is the novel precoding method at the relay. In existing precoding designs, transmitter conducts the precoding to data transmission according to instantaneous channel conditions, e.g., to achieve a high received signal-to-interference plus noise ratio (SINR) [14]. However, in the proposed method, the precoding is designed to improve the channel estimation accuracy as well as simplify the channel estimation processing. The other is the extension of proposed method to MU-MIMO, i.e., the relay assists the uplink data retransmission of multiple UEs to eNodeB. A joint design rule of cyclic shifts and data precoding for multiple transmit antennas at the relay is defined considering cyclic shifts of multiple UEs.

\section{System model}

\section{Uplink transmission/retransmission}

Figure 1 shows the considered system model, where there are in total $M$ UEs in the macro cell and the type II relay is used to assist transmission/retransmission of multiple UEs in the uplink. In the following, we first consider single UE case for discussion simplicity and after that we will extend the discussion to multiple UEs.

The main procedures for type II relay-assisted uplink transmission and retransmission are shown in Figure 2. First, at time $t$, the UE performs initial transmission (data with control information 1 embedded) to the eNodeB. The relay monitors the initial transmission of the UE and detects the initial transmission. Second, if detection of data in initial transmission is not correct at the eNodeB, the eNodeB should reply NACK at time $t+4$. Both the UE and relay monitor the ACK/NACK feedback of the eNodeB at $t+4$ in order to determine whether retransmission is needed or not. If retransmission is needed, the UE should perform retransmission at time $t+8$. In the retransmission, the UE will embed new control information, i.e., control information 2 , if it has any. However, at time $t+8$, the relay cannot know the content of control information 2 to be transmitted by the UE. Therefore, the key issue is how to perform cooperative retransmission by type II relay at time $t+8$ because the relay cannot know control information 2 in advance. If type II relay simply transmits data and control information 1 in the retransmission, the two control information, i.e., control information 2 transmitted by the UE and control information 1 transmitted by the relay, will collide at the eNodeB. The "control information 1" and "control information 2" denote control information transmitted at time $t$ and $t+8$, respectively. The control information can be ACK/NACK of HARQ, rank indicator (RI), periodic CQI report, etc.

One straightforward idea is to perform puncture at the relay in order to avoid the control information collision, i.e., in the retransmission, type II relay only transmits reference signal and data while not control information. However, such a transmission of the relay will lead to channel estimation mismatch for control information detection at the eNodeB. To be more specific, the received reference signal at the eNodeB is a superposed signal from both the UE and relay. Therefore, when eNodeB conducts channel estimation, eNodeB will get a combined channel gain, i.e., sum of UE-to-eNodeB channel and relay-to-eNodeB channel. On the other hand, because control information is only transmitted by the $\mathrm{UE}$, the actual channel gain of control information is the UE-to-eNodeB channel. As the control information is embedded in the data channel, if eNodeB uses the estimated combined channel gain to detect control

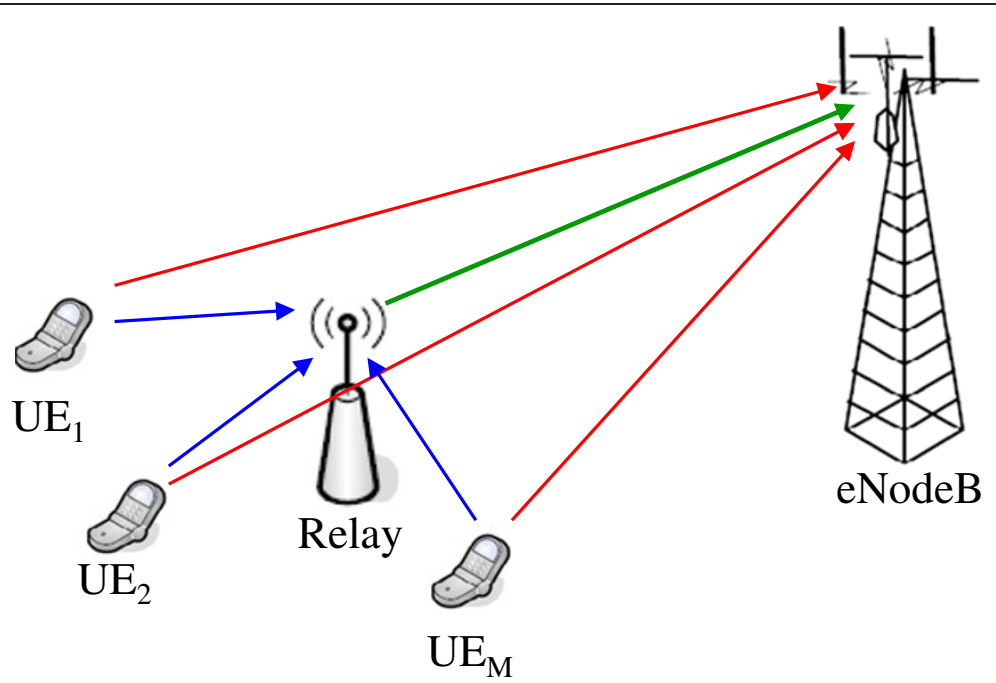

Figure 1 System model. 


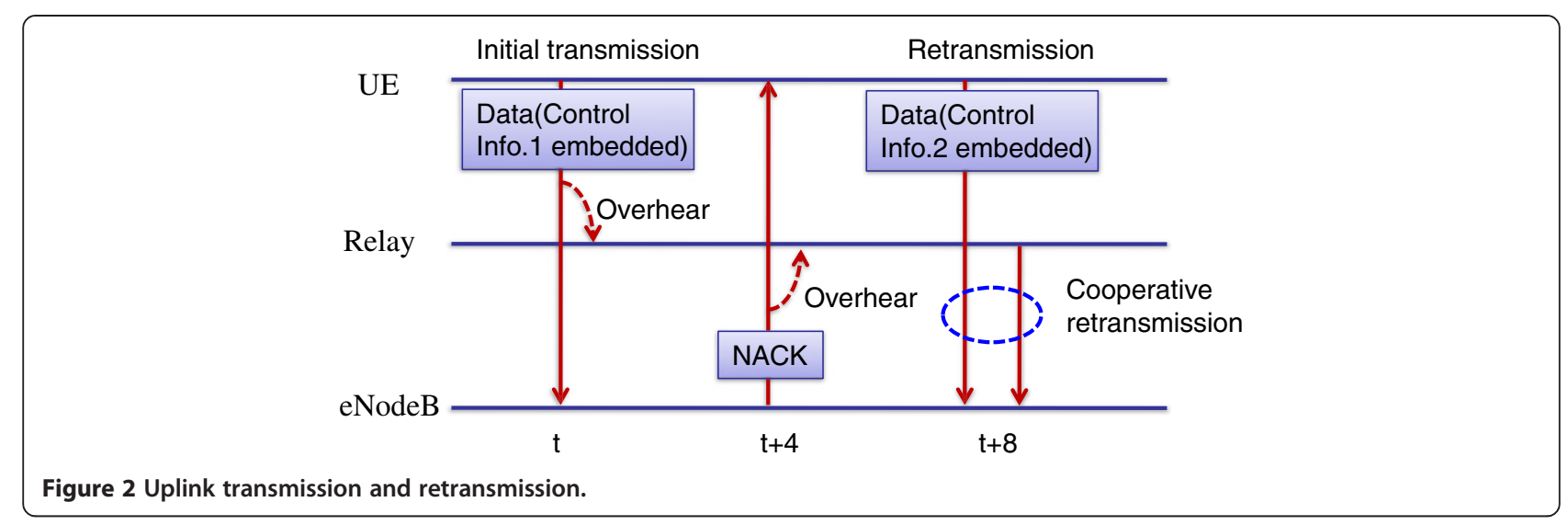

information, the detection performance will be unacceptable due to the mismatched channel gain.

In order to address this channel estimation mismatch problem, some methods have been proposed in $[12,13]$. As discussed in Section 1, although the channel estimation mismatch problem can be solved, the methods in $[12,13]$ have some drawbacks, such as signaling overhead and no cooperative diversity gain. This motives the work in this article. If we smartly design the reference signal and data precoding jointly, the drawbacks of methods in $[12,13]$ can be avoided.

\section{Reference signal in uplink}

Reference signals are defined for uplink channel estimation [15]. The control channel and data channel have their own reference signals. However, if control information is embedded in the data, the eNodeB should use the reference signal of the data channel to detect the embedded control information. The proposed scheme is closely related to the reference signal of data channel, a description of which is given hereafter.

Reference signal sequence $\vartheta_{\mathrm{UE}}(k)$ of the data channel is defined by a cyclic shift $\alpha_{\mathrm{UE}}$ of a base sequence, i.e.,

$$
\vartheta_{U E}(k)=e^{j \alpha_{U E} k} \cdot \bar{\vartheta}(k), \quad 0 \leq k<M_{s c}^{R S},
$$

where $M_{s c}^{R S}$ denotes the number of subcarriers assigned for uplink transmission, $k$ denotes the subcarrier index, and $\bar{\vartheta}(k)$ denotes the base sequence. The cyclic shift $\alpha_{\mathrm{UE}}$ is determined by

$$
\begin{aligned}
& n_{c S}^{U E}=\left(n_{D M R S}^{(1)}+n_{D M R S}^{(2)}+n_{P R S}\right) \bmod 12, \\
& \alpha_{U E}=\frac{2 \pi n_{c s}^{\mathrm{UE}}}{12},
\end{aligned}
$$

where $n_{\mathrm{DMRS}}^{(1)}$ is a value semi-statically configured by higher layer; $n_{\mathrm{DMRS}}^{(2)}$ is dynamically given in the uplink scheduling grant and can take eight values, i.e., $\{0,2,3$, $4,6,8,9,10\}$, and $n_{\mathrm{PRS}}$ is given by a cell-specific pseudorandom sequence. As in (2), the $n_{c s}^{\mathrm{UE}}$ is calculated from three parameters, i.e. $n_{\mathrm{PRS}}, n_{\mathrm{DMRS}}^{(1)}$, and $n_{\mathrm{DMRS}}^{(2)}$. The $n_{\mathrm{PRS}}$ takes fixed cell-specific values. The $n_{\mathrm{DMRS}}^{(1)}$ can be semistatically changed through radio resource control (RRC) signaling by eNodeB and the $n_{\mathrm{DMRS}}^{(2)}$ can dynamically be changed through scheduling grant by eNodeB. Therefore, eNodeB can have very flexible configurations of reference signal sequences used by UEs. In order to reduce signaling overhead for index of reference signal sequences, the $n_{c s}^{\mathrm{UE}}$ is limited to take among 12 candidate values ranging from 0 to 11 as in [15]. After obtaining the $n_{c s}^{\mathrm{UE}}$ according to (2), UE will calculate $\alpha_{\mathrm{UE}}$ in angle domain according to (3) and then reference signal sequence can be generated according to (1). It can be seen that eNodeB knows $\alpha_{\text {UE. }}$. According to (3), the $\alpha_{\mathrm{UE}}$ is calculated from the $n_{c s}^{\mathrm{UE}}$. According to (2), the $n_{c s}^{\mathrm{UE}}$ is calculated from three parameters, i.e., $n_{\mathrm{PRS}}, n_{\mathrm{DMRS}}^{(1)}$, and $n_{\mathrm{DMRS}}^{(2)}$. The $n_{\mathrm{PRS}}$ is calculated from cell ID, eNodeB can know it. The $n_{\text {DMRS }}^{(1)}$ and the $n_{\text {DMRS }}^{(2)}$ are configured by eNodeB through RRC signaling or scheduling grant. So, eNodeB knows the value of $n_{\mathrm{DMRS}}^{(1)}$ and $n_{\mathrm{DMRS}}^{(2)}$. As a result, eNodeB can know the value of the $n_{c s}^{\mathrm{UE}}$ and thus the value of $\alpha_{\mathrm{UE}}$ used by the UE.

\section{Proposed method}

In order to solve the channel estimation mismatch problem and achieve cooperative diversity gain, a novel joint design of reference signal and data precoding used by type II relay for data retransmission is proposed in this section.

\section{Cyclic shift selection of relay reference signal}

In the proposed method, the relay adopts a different reference signal sequence from that of the UE. More specifically, the relay monitors the uplink grant signaling 
transmitted by the eNodeB and obtains the $n_{\mathrm{DMRS}}^{(2)}$ to be used by the UE. Then the relay determines its cyclic shift according to

$$
\begin{aligned}
& n_{\mathrm{cs}}^{\text {Relay }}=\left(n_{D M R S}^{(1)}+n_{D M R S}^{(2)}+\Delta_{\text {offset }}+n_{P R S}\right) \bmod 12, \\
& \alpha_{\text {Relay }}=\frac{2 \pi n_{\mathrm{cs}}^{\text {Relay }}}{12}
\end{aligned}
$$

where $n_{c s}^{\text {Relay }}$ denotes an intermediate parameter (taking value of integer ranging from 0 to 11), which is used to calculate cyclic shift $\alpha_{\text {Relay }}$ in angle domain for reference signal sequence generation by the relay, $\Delta_{\text {offset }}$ denotes a non-zero offset and its value can be optimized to maximize the cyclic shift distance between the UE and the relay in order to obtain the best channel estimation performance. With the $n_{c s}^{\text {Relay }}$, the $\alpha_{\text {Relay }}$ can be calculated according to (5). In order to save signaling overhead to configure these parameters for relay, the relay reuses these parameters of the UE, i.e., the relay adopts the same parameters $n_{\mathrm{PRS}}, n_{\mathrm{DMRS}}^{(1)}$, and $n_{\mathrm{DMRS}}^{(2)}$ as the UE in existing methods [12,13]. However, in the proposed method we propose to introduce an additional non-zero offset, i.e. $\Delta_{\text {offset }}$, in calculation of the $n_{c s}^{\text {Relay }}$. With this non-zero offset, the $n_{c s}^{\text {Relay }}$ will always take different values from the $n_{c s}^{\mathrm{UE}}$. Therefore, the relay will always generate a different reference signal sequence from that generated by the UE. For example,

$$
\Delta_{\text {offset }}^{\text {Optimal }}=\underset{\Delta_{\text {offset }}}{\arg \max }\left|\alpha_{\text {Relay }}-\alpha_{U E}\right| .
$$

The reference signal sequence used by the relay can be calculated as

$$
\vartheta_{\text {Relay }}(k)=e^{j \alpha_{\text {Relay }} k} \cdot \bar{\vartheta}(k) .
$$

Rule for the relay to choose the value of $\Delta_{\text {offset }}$ can be predefined at the eNodeB and relay. Thus, the eNodeB can know reference signal sequence $\vartheta_{\text {Relay }}(k)$ used by the relay without any additional signaling.

Because different cyclic shifts are used by the relay and UE, the eNodeB can estimate the channel response of the control information transmitted in the data channel from the UE to the eNodeB on the basis of the cyclic shift of the UE even though the reference signals of the UE and relay are superposed at the eNodeB. To be more specific, if eNodeB performs fast Fourier transform to the overlapped reference signals, the two channel responses, i.e., UE-to-eNodeB channel and relay-to-eNodeB channel, will have different locations in time domain due to the different cyclic shifts. Therefore, eNodeB can filter out the reference signal of relay in time domain (e.g., using the method in [16]) and thus can have an accurate estimation of the UE-to-eNodeB channel. Because the actual channel of the control information transmission is the UE-to-eNodeB channel, eNodeB can conduct detection of the control information with the estimated channel. Therefore, the channel estimation mismatch problem can effectively be solved.

\section{Precoding for data retransmission}

After determining the cyclic shift for the reference signal, the relay performs precoding to the data according to its own cyclic shift and that of the UE. Purpose of the precoding is to simplify channel estimation at the eNodeB as well as to improve the channel estimation accuracy. The data transmitted by the relay is

$$
\begin{aligned}
& d_{\text {Relay }}(k)=W(k) \cdot d(k), \\
& W(k)=e^{j\left(\alpha_{\text {Relay }}-\alpha_{U E}\right) k},
\end{aligned}
$$

where $W(k)$ denotes the proposed precoding and $d(k)$ denotes data for re-transmission. From (8) and (9), it can be seen that the proposed precoding only changes the phase of the data in the frequency domain, which equals a shift of the data in the time domain. Therefore, the proposed precoding does not change the PAPR of the transmitted signal.

For cooperative retransmission at time $t+8$, the reference signal, data, and control information transmitted by the UE are

$$
\begin{aligned}
& \vartheta_{U E}(k)=e^{j \alpha_{U E} k} \cdot \bar{\vartheta}(k), \\
& d_{\mathrm{UE}}(k)=d(k), \\
& s_{U E}(k)=s(k),
\end{aligned}
$$

where $s(k)$ denotes modulated symbols of the control information. The reference signal, data, and control information transmitted by the relay are

$$
\begin{aligned}
& \vartheta_{\text {Relay }}(k)=e^{j \alpha_{\text {Relay }} k} \cdot \bar{\vartheta}(k), \\
& d_{\text {Relay }}(k)=e^{j\left(\alpha_{\text {Relay }}-\alpha_{U E}\right) k} \cdot d(k), \\
& s_{\text {Relay }}(k)=0 .
\end{aligned}
$$

Therefore, the received reference signal, data, and control information at the eNodeB are

$$
\begin{aligned}
\vartheta_{e N B}(k) & =H_{M B}(k) \cdot \vartheta_{U E}(k)+H_{R B}(k) \cdot \vartheta_{\text {Relay }}(k)+n_{1} \\
& =H_{M B}(k) \cdot e^{j \alpha_{u E} k} \cdot \bar{\vartheta}(k)+H_{R B}(k) \cdot e^{\alpha_{\text {Relay }} k} \cdot \bar{\vartheta}(k)+n_{1},
\end{aligned}
$$




$$
\begin{aligned}
d_{e N B}(k) & =H_{M B}(k) \cdot d_{\mathrm{UE}}(k)+H_{R B}(k) \cdot d_{\text {Relay }}(k)+n_{2} \\
& =\left(H_{M B}(k)+H_{R B}(k) \cdot e^{j\left(\alpha_{\text {Relay }}-\alpha_{U E}\right) k}\right) \cdot d(k)+n_{2},
\end{aligned}
$$

where $H_{\mathrm{MB}}(k)$ and $H_{\mathrm{RB}}(k)$ denote the channel response from the UE to the eNodeB and from the relay to the eNodeB on the $k$ th subcarrier, respectively. $n_{1}, n_{2}$, and $n_{3}$ denote the noise.

For data detection, the eNodeB first performs channel estimation of the data channel through the received reference signal. We can simply re-write (16) as

$$
\begin{aligned}
\vartheta_{\mathrm{eNB}}(k)= & \underbrace{\left(H_{\mathrm{MB}}(k)+H_{\mathrm{RB}}(k) \cdot e^{j\left(\alpha_{\mathrm{Relay}}-\alpha_{\mathrm{UE}}\right) k}\right)}_{\text {Equivalent channel }} \\
& \times \underbrace{e^{j \alpha_{\mathrm{UE}} k} \cdot \bar{\vartheta}(k)}_{\text {Reference signal of UE }}+n_{1} .
\end{aligned}
$$

From (19), the eNodeB can simply perform channel estimation of the data channel using the reference signal of the UE. Then the eNodeB can obtain an equivalent channel. From (17), we can see that the equivalent channel is just the same as the equivalent channel of the data transmission because of the proposed precoding. Therefore, the eNodeB can simply use the estimated equivalent channel for data detection.

If the proposed precoding is not used, the received data at the eNodeB is

$$
\begin{aligned}
d^{\prime}{ }_{\mathrm{eNB}}(k) & =H_{\mathrm{MB}}(k) \cdot d_{\mathrm{UE}}(k)+H_{\mathrm{RB}}(k) \cdot d_{\mathrm{Relay}}(k)+n_{2} \\
& =\underbrace{\left(H_{\mathrm{MB}}(k)+H_{\mathrm{RB}}(k)\right)}_{\text {Equivalent channel }} \cdot d(k)+n_{2}
\end{aligned}
$$

Therefore, the eNodeB has to separately estimate $H_{\mathrm{MB}}(k)$ and $H_{\mathrm{RB}}(k)$ from (16) and then calculates the equivalent channel of the data transmission according to $(20)$.

It can be seen that the proposed precoding not only simplifies the channel estimation operation, but also improves the channel estimation accuracy. If the eNodeB performs the channel estimation of $H_{\mathrm{MB}}(k)$ and $H_{\mathrm{RB}}(k)$ separately, the SINR for the channel estimation is low. For example, if the eNodeB estimates the channel from the UE to eNodeB, signal from the relay to eNodeB is regarded as interference. However, in the proposed method, the eNodeB directly estimates the superposed equivalent channel. Because the superposed channel has a high SINR for channel estimation, the channel estimation accuracy is improved.

For control information detection, the eNodeB estimates $H_{\mathrm{MB}}(k)$ from (16) by filtering out the reference signal of the relay in the time domain, e.g., using the method in [16], and then uses the estimated channel for control information detection according to (18).

The multiplexing method of control information and data is conducted according to [17]. According to the number of bits of the control information, UE will conduct rate matching to generate data bits to be multiplexed with the control information. If the new control information has different bit number to the old control information, rate matching result of data bits will change accordingly. Although the relay does not know the content of the new control information, the relay does know which kind of control information will be transmitted by the UE, e.g., periodic CQI, ACK/NACK of HARQ. Therefore, the relay can know bit number of the new control information and thus can perform the same rate matching as the UE to generate the data bits. Because the mapping rule of data bits to resource is predefined, the UE and the relay will have the same mapping results for data transmission. As a result, even if the new control information has different bit number compared with the old control information, the proposed method can still be applied.

In the proposed method, we assume the relay has detection function and CRC check function. During the initial transmission of UE, the relay conducts data detection and CRC check. If the CRC check is successful, the relay can know the data transmitted by the UE. If the CRC check is failed, the relay will not conduct the retransmission and the UE will perform the retransmission by itself. It is noted that there is no need to inform eNodeB about whether the relay conducts retransmission or not. eNodeB can always use the same channel estimation algorithm (e.g., algorithm in [16]) to estimate the channel response for control information detection, i.e., eNodeB performs filtering to location of reference signal of relay in time domain even if relay does not perform the retransmission. Therefore, regardless the relay conducts retransmission or not, eNodeB can always get correct channel estimation for control information detection and the proposed method does not introduce any additional signaling overhead.

\section{Extension to MU-MIMO}

The proposed scheme can be extended to MU-MIMO, i.e., the relay assists the data retransmission of multiple users to the eNodeB. We assume that there are $M$ UEs with single transmit antenna. The eNodeB has $N(N \geq M)$ receive antennas. The relay has $P(P \geq M)$ receive antennas and $\mathcal{Q}(\mathcal{Q} \geq 1)$ transmit antennas.

In data retransmission, the reference signal, data, and control information transmitted by the UE $m(1 \leq m \leq M)$ are

$$
\begin{aligned}
& \vartheta_{U E}^{m}(k)=e^{j \alpha_{U E}^{m} k} \cdot \bar{\vartheta}(k), \\
& d_{\mathrm{UE}}^{m}(k)=d_{m}(k),
\end{aligned}
$$




$$
s_{U E}^{m}(k)=s_{m}(k)
$$

The reference signal, data, and control information transmitted by antenna $q(1 \leq q \leq \mathcal{Q})$ of the relay are

$$
\begin{aligned}
& \theta_{\text {Relay }}^{q}(k)=\sum_{m=1}^{M} e^{j\left(\Delta_{\text {Relay }}+\alpha_{\mathrm{UE}}^{m}\right) k} \cdot \bar{\vartheta}(k), \\
& d_{\text {Relay }}^{q}(k)=\sum_{m=1}^{M} e^{j \Delta_{\text {Relay }} k} \cdot d_{m}(k), \\
& s_{\text {Relay }}^{q}(k)=0
\end{aligned}
$$

where $\Delta_{\text {Relay }}$ denotes an incremental cyclic shift used by the relay.

Therefore, the received reference signal, data, and control information at receive antenna $n(1 \leq n \leq N)$ at the eNodeB are

$$
\begin{aligned}
\vartheta_{\mathrm{eNB}}^{n}(k)= & \sum_{m=1}^{M} h_{n m}(k) \cdot e^{j \alpha_{\mathrm{UE}}^{m} k} \cdot \bar{\vartheta}(k) \\
& +\frac{1}{\sqrt{Q}} \sum_{q=1}^{Q} h_{n q}(k) \cdot \sum_{m=1}^{M} e^{j\left(\Delta_{\text {Relay }}+\alpha_{\mathrm{UE}}^{m}\right) k} \cdot \bar{\vartheta}(k) \\
& +n_{4}, \\
d_{\mathrm{eNB}}^{n}(k)= & \sum_{m=1}^{M} h_{n m}(k) \cdot d_{m}(k)+\frac{1}{\sqrt{Q}} \sum_{q=1}^{Q} h_{n q}(k) \\
& \times \sum_{m=1}^{M} e^{j \Delta_{\mathrm{Relay}} k} \cdot d_{m}(k)+n_{5} \\
= & \sum_{m=1}^{M} \underbrace{\left(h_{n m}(k)+\frac{1}{\sqrt{Q}} \sum_{q=1}^{Q} h_{n q}(k) e^{j \Delta_{\text {Relay }} k}\right)}_{\text {Equivalent channel for UE } m} \\
& \times d_{m}(k)+n_{5}, \\
s_{\mathrm{eNB}}^{n}(k)= & \sum_{m=1}^{M} h_{n m}(k) \cdot s_{m}(k)+n_{6},
\end{aligned}
$$

where $h_{n m}(k)$ and $h_{n q}(k)$ denote the channel response from receive antenna $n$ of the eNodeB to the UE $m$ and transmit antenna $q$ of the relay on the $k$ th subcarrier, respectively.

We rewrite (27) as

$$
\begin{aligned}
\vartheta_{\mathrm{eNB}}^{n}(k)= & \sum_{m=1}^{M}\left(h_{n m}(k)+\frac{1}{\sqrt{Q}} \sum_{q=1}^{Q} h_{n q}(k) \cdot e^{j \Delta_{\mathrm{Relay}} k}\right) \\
& \times e^{j \alpha_{\mathrm{UE}}^{m} k} \cdot \bar{\vartheta}(k)+n_{4} .
\end{aligned}
$$

From (28) and (30), eNodeB can perform channel estimation to obtain the superposed equivalent channel of data transmission of the UE $m$, e.g., using the method in [16]. After obtaining the equivalent channels (in total $N \times M$ ) of all UEs, eNodeB can construct an equivalent MIMO channel matrix and perform MIMO detection to detect the data packets of $M$ UEs.

Two kinds of diversity gain will be achieved when the proposed method is used. One is time-domain diversity gain due to HARQ combining of initial transmission and retransmission. The other is cooperative diversity gain enabled by the proposed precoding method in retransmission by UE and type II relay.

\section{Performance evaluation}

We assume MU-MIMO with two single antenna UEs and the numbers of antennas for the eNodeB and relay are two. The 6-ray typical urban channel model with the r.m.s. delay spread of $1.06 \mu \mathrm{s}$ [18] is used for both direct link (UE-to-eNodeB) and backhaul link (relay-to-eNodeB). The resource block has a bandwidth of $180 \mathrm{kHz}$ and the number of resource blocks assigned for data transmission and control information transmission is 10 and 1 , respectively. The modulation is 16QAM for data transmission and QPSK for control information transmission. Turbo code is used for data transmission and tailed convolutional code is used for control information transmission. The coding rate is one half or one-third for both the data transmission and control information transmission. Two kinds of cyclic shift selection are considered for the proposed method: one is random selection without optimization and the other is optimized selection that maximizes the cyclic shift distance among UEs and the relay. The channel estimation algorithm in [16] is used for both data and control information. The Doppler frequency used in the simulations is set to $10 \mathrm{~Hz}$. The performances of the relay puncture, i.e., relay punctures the control information in the data retransmission, the method in [12] and the method in [13] are also simulated as references.

Figure 3 shows the BLER of the control information transmission of each retransmission. It can be seen that (1) a simple puncture at relay exhibits a very poor performance due to the mismatched channel estimation at the eNodeB. The BLER performance is as worse as $7 \times 10^{-1}$ even at very high SNR, which is unacceptable for a practical system. (2) The method in [13] has the best performance under real channel estimation assumption because only UE transmits reference signal and control information, i.e., there is no channel estimation mismatch problem for control information and there is no interference from the relay for reference signal. For the method in [12], the performance of control information is not shown because the control information is 


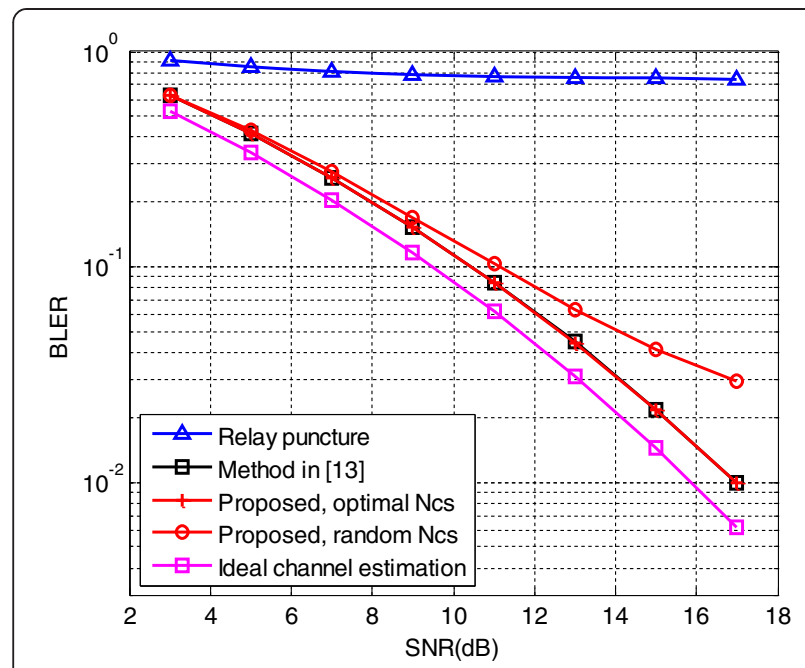

Figure 3 BLER performance of control information: code rate = $1 / 2$, QPSK.

transmitted in control channel. The control channel has different transmission processing, e.g., spreading in time domain, compared with data channel and thus the performance of method in [12] is not shown. (3) The proposed scheme with optimized cyclic selection and the method in [13] achieve the same performance, which means that the maximum distance cyclic shift selection is very effective and enables very accurate channel estimation of $H_{\mathrm{MB}}(k)$ from the superposed reference signals. (4) The optimized cyclic shift selection achieves better performance than the random cyclic shift selection due to its higher channel estimation accuracy. As a conclusion, the proposed method can achieve the same performance for control information as the method in [13] with proper cyclic shift selection.

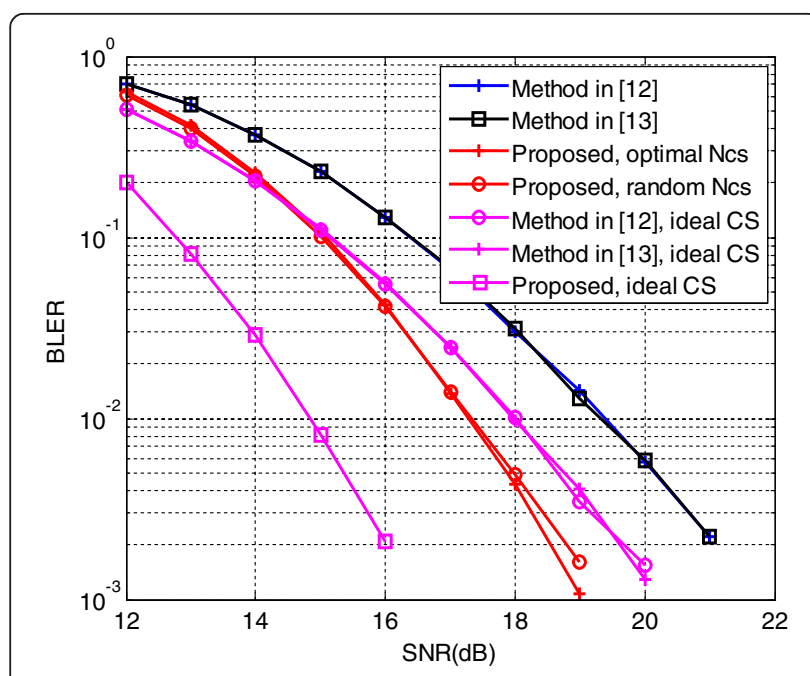

Figure 4 BLER performance of data: code rate $=1 / 2,16 Q A M$.

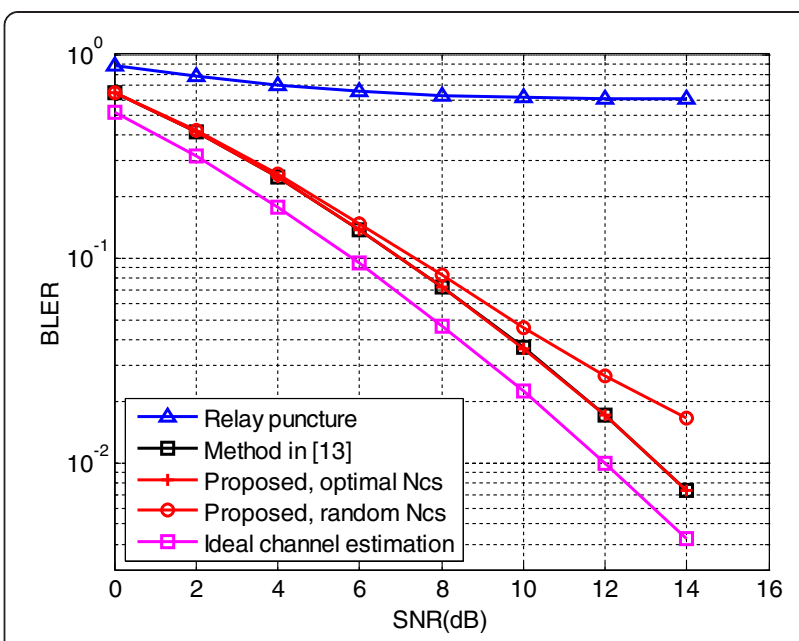

Figure 5 BLER performance of control information: code rate $=$ $1 / 3$, QPSK.

Figure 4 shows the BLER of each data retransmission. The simulation results can be divided into two groups. One is performance comparison with ideal channel estimation and the other is performance comparison with real channel estimation. From the simulation results, it can be seen that (1) the proposed method can achieve 2-3 dB performance improvement compared with the methods in $[12,13]$ for both ideal channel estimation and real channel estimation due to the cooperative diversity gain. (2) The proposed method with real channel estimation performs even better than the methods in $[12,13]$ with ideal channel estimation when SNR is high, e.g., when SNR is larger than $15 \mathrm{~dB}$. This is because with the increase of SNR, the accuracy of real channel estimation increases and thus the impact of channel estimation on performance decreases. Therefore, the proposed method with real channel estimation can even achieve a

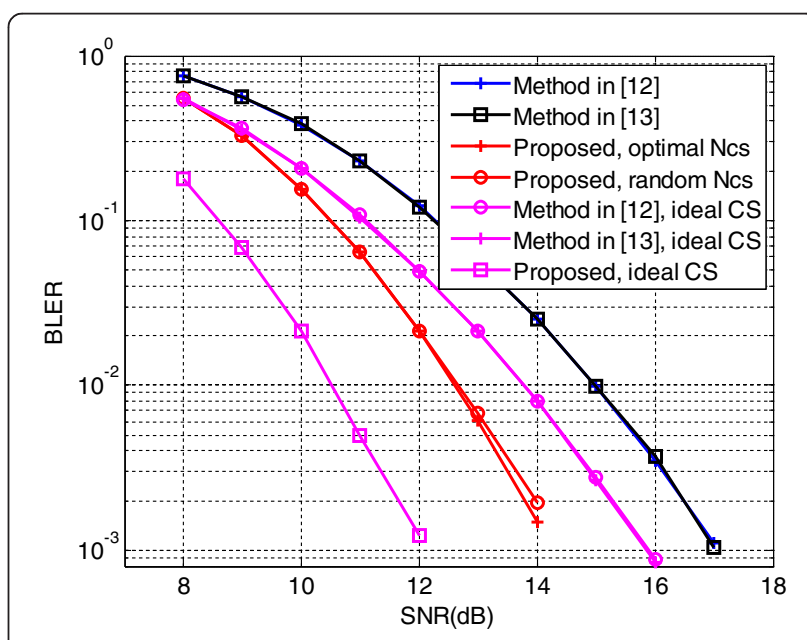

Figure 6 BLER performance of data: code rate $=1 / 3,16 Q A M$. 
better performance than the methods in [12,13] with ideal channel estimation. As a conclusion, the proposed method can achieve better performance for data than the methods in $[12,13]$.

Figures 5 and 6 show BLER comparisons of control information and data, respectively, when code rate equals to $1 / 3$. When code rate reduces, performances of all the schemes improve as expected. For control information, similar conclusions can be drawn that (1) the "relay puncture" cannot work even if the code rate is reduced although the performance becomes a little better than that when code rate equals to $1 / 2$. (2) The proposed scheme with optimized cyclic shift selection and the method in [13] achieve the same performance. (3) The optimized cyclic shift selection achieves better performance than the random cyclic shift selection as expected. For performance comparison of data, it can be seen that the proposed method can achieve a performance improvement of $2-3 \mathrm{~dB}$ over the methods in [12,13] because of the cooperative diversity gain.

In summary, simulation results show that the proposed method can achieve good performance for both control information and data compared with existing methods, and thus is an effective method for type II relay system.

\section{Conclusions}

A novel relay-assisted retransmission scheme is proposed for the uplink of LTE-Advanced systems. First, a novel joint design of reference signal and data precoding at the relay are proposed, where the data precoding is conducted on the basis of the cyclic shift of the reference signal. By such a joint design, the channel estimation mismatch problem of control information is solved and cooperative diversity gain is achieved for data retransmission. Second, the proposed design is further extended to support MU-MIMO, i.e., the relay assists the uplink data retransmission of multiple UEs to eNodeB. A joint design rule of cyclic shifts and data precoding for multiple transmit antennas at the relay is proposed considering cyclic shifts of multiple UEs. Simulations are conducted to verify the effectiveness of the proposed scheme. From the simulation results, the mismatched channel estimation leads to very bad performance of the control information and simple puncture at the relay can not work even at high SNR. The proposed scheme is very effective, which achieves better performance than existing schemes for different coding rate and modulation order. Optimized cyclic shift selection at the relay can achieve better performance than random cyclic shift selection, but the gain is decreased for MU-MIMO. evolution; PAPR: peak-to-average power ratio; SINR: signal-to-interference plus noise ratio; UE: user equipment.

\section{Competing interests}

The authors declare that they have no competing interests.

\section{Author details}

${ }^{1}$ DOCOMO Beijing Communications Laboratories Co, Ltd, 7/F, Raycom Infotech Park Tower A, No. 2 Kexueyuan South Road, Haidian District, Beijing 100190, China. ${ }^{2}$ Radio Access Network Development Department, NTT DOCOMO, INC., 3-5 Hikarino-oka, Yokosuka-shi, Kanagawa-ken 239-8536, Japan.

Received: 19 June 2012 Accepted: 20 February 2013

Published: 23 March 2013

\section{References}

1. D Soldani, S Dixit, Wireless relays for broadband access. IEEE Commun. Mag. 46(3), 58-66 (2008)

2. G Liebl, T Moraes, P Weitkemper, Advanced Relay Technical Proposals (2nd edn, ARTIST4G project deliverables, 2011, 2011)

3. A Papadogiannis, M Farber, A Saadani, MD Nisar, P Weitkemper, Y Sui, T Svensson, D Ktenas, N Cassiau, TMD Moraes, Advanced relaying concepts for future wireless networks, in Future Network \& Mobile Summit. Conference Proceedings: Berlin 2012, 1-10 (2012)

4. R Hoshyar, R Tafazolli, Performance evaluation of HARQ schemes for cooperative regenerative relaying, in IEEE International Conference on Communications. Dresden, 1-6 (2009)

5. Y Yang, H Hu, J Xu, G Mao, Relay technologies for WiMax and LTE-advanced mobile systems. IEEE Commun. Mag. 47(10), 100-105 (2009)

6. Y Zhu, W Lin, X Yang, A novel downlink adaption scheme for TDD type-II relay systems, in International Conference on Measuring Technology and Mechatronics Automation. Shanghai, 191-194 (2011)

7. K Loa, C-C Wu, S-T Sheu, Y Yuan, M Chion, D Huo, L Xu, IMT-advanced relay standards. IEEE Commun. Mag. 48(8), 40-48 (2010)

8. 3GPP, Further advancements for E-UTRA physical layer aspects (TR 36814, V900th edn, 2010)

9. 3GPP, Alcatel-Lucent, R1-100951st edn (Alcatel-Lucent Shanghai Bell and CHTTL, Type 2 relay summary, 2010, 2010)

10. 3GPP, Qualcomm Europe, R1-093113th edn (Challenges with type II relay operation, 2009, 2009)

11. 3GPP, Characteristics, implications, and assessment of type-ll relaying, 2009 (Ericsson, R1-092025th edn, 2009)

12. 3GPP, LG Electronics (R1-100237th edn, PUSCH forwarding in type II relay, 2010, 2010))

13. 3GPP, Fujitsu (R1-100584th edn, Considerations on using type 2 relay for UL transmission, 2010, 2010)

14. Q Li, RQ Hu, Q Yi, G Wu, Cooperative communications for wireless networks: techniques and applications in LTE-advanced systems. IEEE Wirel. Commun. 19(2), 22-29 (2012)

15. 3GPP, Evolved Universal Terrestrial Radio Access (E-UTRA) (TS 36211, v1010th edn, physical channels and modulation, 2011, 2011)

16. X Hou, Z Zhang, H Kayama, DMRS design and channel estimation for LTEadvanced MIMO uplink, in IEEE VTC2009-Fall (Anchorage, 2009), pp. 1-5

17. 3GPP, Evolved Universal Terrestrial Radio Access (E-UTRA) (TS 36212, v1030th edn, multiplexing and channel coding, 2011, 2011)

18. 3GPP, Physical layer aspects for Evolved UTRA (TR 25814, V710th edn, 2006)

doi:10.1186/1687-6180-2013-57

Cite this article as: Li et al:: A novel type II relay-assisted retransmission scheme for uplink of LTE-advanced system. EURASIP Journal on Advances in Signal Processing 2013 2013:57. 